\title{
Stage II pressure ulcer caused by the connection of an electrocardiogram's nonstandard probe on the patient's chest: A case report study
}

\section{Hossein Rafiei}

Social Determinants of Health Research Center, Research Institute for prevention of NonCommunicable Diseases, Qazvin University of Medical Sciences, Qazvin, Iran.

Corresponding author: Hossein Rafiei, Social Determinants of Health Research Center, Qazvin University of Medical Sciences, Qazvin, Iran. E-mail: h.rafiei@qums.ac.ir

Cite this article: Rafiei H. Stage II pressure ulcer caused by the connection of an electrocardiogram's nonstandard probe on the patient's chest: A case report study. Int J Epidemiol Health Sci 2020;1(1): e01. Doi:10.51757/IJEHS.1.1.2020.39686.

\begin{abstract}
The occurrence of pressure ulcers can be problematic for patients and health care systems. A medical device-related pressure ulcer (MDRPU) is considered as one of the important indicators of patient safety assessment as well as the quality of care provided to the patient. This case report concerns a 54-year-old male patient, who was admitted to the cardiac unit of a hospital. The patient developed stage II pressure ulcers due to the connection of a nonstandard probe of the electrocardiogram to its chest. Nurses had not taken any action to treat the patient's ulcers. To improve this matter, necessary measures such as training courses should be considered by the officials and healthcare planners. Apart from this, to prevent the development of MDRPUs in the future, it is of great importance to pay special attention to the probes that are being used for the recording of an ECG.
\end{abstract}

Keywords: Medical device-related pressure ulcer, electrocardiogram probe, Stage II

\section{Introduction}

The occurrence of pressure ulcers can be associated with various problems for the patient and the healthcare providers such as the experience of pain, loss of autonomy, decreased quality of life, increased length of hospital stay, increased risk of infections and an increased mortality rate (1-5). Furthermore, pressure ulcers are associated with high costs. In 2019, a study conducted in Iran reported that the treatment- related costs of pressure ulcers are between $\$ 12$ and $\$ 66000$ for each ulcer (6).

According to the definition given by the National Pressure Ulcer Advisory Panel (NPUAP), a pressure ulcer is "localized damage to the skin and/or underlying soft tissue usually over a bony prominence or related to a medical or another device as a result of excessive pressure" (7). As the definition of a pressure ulcer implies, the occurrence of such damages can 
sometimes be attributed to medical devices that are applied on the patient's body. Medical device-related pressure ulcer (MDRPU) is considered as one of the important indicators of patient safety assessment as well as the quality of care provided to the patient (1). MDRPU is defined as an ulcer caused by the application of a medical device, whereby the damage is beneath the area of the device and its shape on the patient's skin is usually in accordance with the shape of the device (8).

There can be several reasons for a pressure ulcer to manifest following the application of medical devices. Medical devices are usually hard and non-elastic, which after use, may cause excessive pressure on the skin under the device. On the other hand, in order to provide maximum performance, medical devices should be firmly fixed at the time of use. This might cause excessive pressure on the patient's skin under the device and can thereby result in a pressure ulcer. The process of skin damage can be accelerated by

\section{Case report}

A 54-year-old male patient presented with chest pain and diagnosed with myocardial infarction was hospitalized in the cardiology ward. The patient's vital signs, including his blood pressure, heart rate and respiratory rate were in the normal range. The drugs the patient used, included Metoral, Nitrocontin, Captopril, Pantoprazole, Aspirin, Atorvastatin, and Plavix. To further assess the patient's condition, a daily ECG was ordered for him. He was then transferred to the heart center of the province after being hospitalized in a hospital in the suburb to receive factors such as excessive moisture and the high temperature of the skin under the medical device (9). In a review study conducted in 2019 , the prevalence and incidence of such damages in hospital setting was reported to be $10 \%$ and $12 \%$ respectively (1). In another study, Black e al. reported that the risk of pressure ulcer development in patients attached with medical devices is 2.4 times higher compared with patients without any medical device attached to them (9). Given the importance of this issue, the attention of the prevention and care team to such damages has increased in recent years and we are witnessing a rise in the number of articles in this regard. Such damage can be caused by inappropriate use of devices including cervical collars, oxygen masks, splints, vein catheters, tracheal tubes, tracheostomy tubes, pulse oximeters and nasogastric tubes (9-13). In the present case study, a case of pressure ulcer related to the use of a nonstandard probe of an electrocardiogram (ECG) will be reported.

advanced treatment. After carrying out an ECG, the cardiac nurse noticed an ulcer on the patient's chest. The ulcer was on the left side of the sternum area and below the nipple. Careful examination showed that the damage was caused by excessive pressure related to the connection of the probe of the ECG to the patient's skin during the recording of the ECG. A thorough examination of the skin revealed two pressure ulcers, which both were in stage II (figure 1). Surprisingly, the nurses had not taken any action to treat the injuries. 


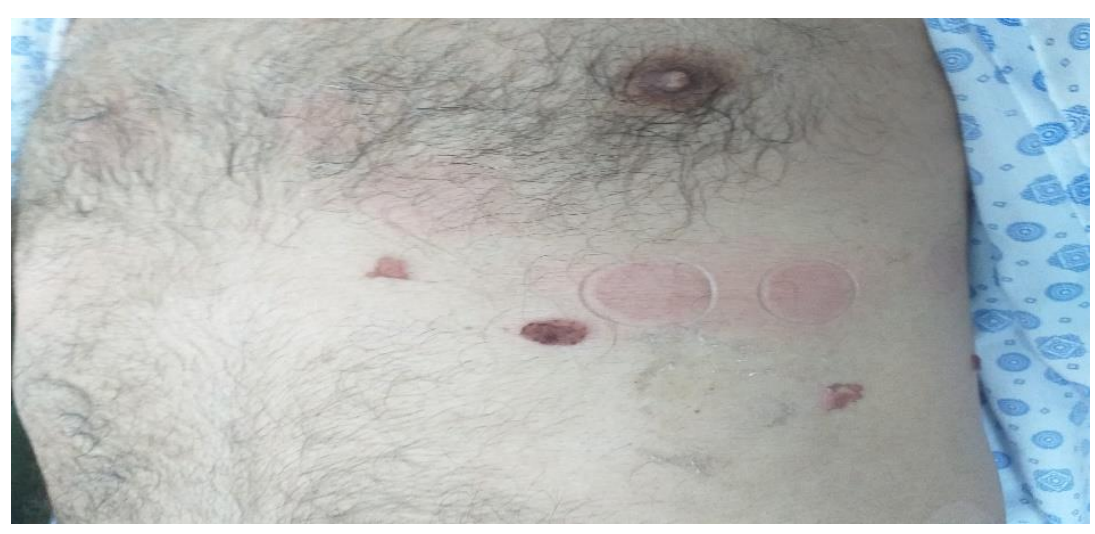

Figure 1. Development of pressure ulcers due to the connection of a nonstandard probe of the ECG to the patient's chest.

\section{Discussion}

Case studies have shown the occurrence of MDRPUs in different areas of the body such as occiput, chin, mouth, neck, heel, toe, nose and buttocks (9-12). To date, however, there has been no case of pressure ulcer related to the inappropriate use of the probe of the ECG.

Our searches only showed one study in Iran that examines the incidence and risk factors of MDRPUs (13). Therefore, considering the numerous cases reported on MDRPU in Iran, further study in this regard is required. While aiding patients attached to medical devices, nurses should pay special attention to the pressure these devices may have on one's skin. It seems that in the examined patient, the probe used in the ECG was not standard and put excessive pressure on his skin. The surprising point of this case was the lack of action by nurses to deal with the pressure ulcers after their occurrence. It seems that Iranian nurses do not have the required information regarding MDRPU. To improve this matter, necessary measures such as training courses should be considered by the officials and healthcare planners. Using guidelines developed by NPUAP that have 7 recommendations, can also be beneficial for the prevention of medical device-related pressure ulcers in clinical areas (7).

One of the differences between MDRPUs and regular pressure ulcers is that there is no possible way to predict the risk of such damages by using a criterion, such as the Braden Scale for Predicting Pressure Ulcer Risk. For instance, if the Braden Scale was used for the studied patient in this case report, the patient would have a low risk for pressure ulcer development. Therefore, it is necessary to design a more optimized scale for predicting the risk of MDRPUs. Apart from this, it is important to bear in mind that the use of a nonstandard probe might result in skin damage and can thereby lead to the development of MDRPUs. To prevent such incidents in the future, it is of great importance to pay special attention to the probes that are being used for the recording of an ECG. 


\section{References}

1. Jackson D., Sarki A.M., Betteridge R. and Brooke J. Medical device-related pressure ulcers: A systematic review and meta-analysis. Int J Nurs Stud. 2019; 92:109-20.

2. Kayser S.A., VanGilder C.A., Ayello E.A. and Lachenbruch C. Prevalence and Analysis of Medical Device-Related Pressure Injuries: Results from the International Pressure Ulcer

Prevalence Survey. Adv Skin Wound Care. 2018; 31(6):276-285.

3. Mehta C., Ali M., Mehta Y., George J.V. and Singh M.K. MDRPU-an uncommonly recognized common problem in ICU: A point prevalence study. J Tissue Viability. 2019; 28(1):35-9.

4. Barakat-Johnson M., Barnett C., Wand T. and White K. Medical device-related pressure injuries: An exploratory descriptive study in an acute tertiary hospital in Australia. J Tissue Viability. 2017; 26(4):246-53.

5. Iranmanesh S., Rafiei H. and Esmaeili Abdar M. Letter: A case of pressure ulcer development on a patient's ear as a result of pulse oximetry probe. Int Wound J. 2012; 9(6):701-2.

6. Zarei E., Madarshahian E., Nikkhah A. and Khodakarim S. Incidence of pressure ulcers in intensive care units and direct costs of treatment: Evidence from Iran. J Tissue Viability. 2019; 28(2):70-4

7. National Pressure Ulcer Advisory Panel, European Pressure Ulcer Advisory Panel and Pan Pacific
Pressure Ulcer Alliance. Prevention and Treatment of Pressure Ulcers: Quick Reference Guide. Emily Haesler (Ed.). Cambridge Media: Perth, Australia; 2014.

8. Makic M.B. Medical Device-Related Pressure Ulcers and Intensive Care Patients. J Perianesth Nurs 2015; 30(4):336-7.

9. Black J.M., Cuddigan J.E., Walko M.A., Didier L.A., Lander M.J. and Kelpe M.R. Medical device related pressure ulcers in hospitalized patients. Int Wound J. 2010; 7(5):358-65.

10. Barakat-Johnson M., Barnett C., Wand T. and White K. Medical device-related pressure injuries: An exploratory descriptive study in an acute tertiary hospital in Australia. J Tissue Viability. 2017; 26(4):246-53.

11. Black J., Alves P., Brindle C.T., Dealey C., Santamaria N., Call E. and Clark M. Use of wound dressings to enhance prevention of pressure ulcers caused by medical devices. Int Wound J. 2015; 12(3):322-7.

12. Kim J.Y., Lee Y.J. and Korean Association of Wound Ostomy Continence Nurses. Medical devicerelated pressure ulcer (MDRPU) in acute care hospitals and its perceived importance and prevention performance by clinical nurses. Int Wound J. 2019;16 (Suppl 1):51-61.

13. Rashvand F., Shamekhi L., Rafiei H. and Nosrataghaei M. Incidence and risk factors for medical device-related pressure ulcers: The first report in this regard in Iran. Int Wound J. 2020; 17(2):436442. 